\title{
Arquitectura de tierra: el adobe como material de construcción en la época prehispánica
}

\author{
Jorge E Gama-Castro 1,*, Tamara Cruz y Cruz ${ }^{2}$, Teresa Pi-Puig ${ }^{1}$, René Alcalá-Martínez ${ }^{1}$, \\ Héctor Cabadas-Báez ${ }^{2}$, Carolina Jasso-Castañeda ${ }^{3}$, Jaime Díaz-Ortega ${ }^{1}$, \\ Serafín Sánchez-Pérez ${ }^{2}$, Fernando López-Aguilar ${ }^{4}$, Rodrigo Vilanova de Allende ${ }^{4}$ \\ ${ }^{1}$ Instituto de Geología, Universidad Nacional Autónoma de México, Ciudad Universitaria, 04510, México, D.F. \\ ${ }^{2}$ Posgrado en Ciencias de la Tierra, Instituto de Geología, Universidad Nacional Autónoma de México, Ciudad Universitaria, 04510, \\ México, D.F. \\ ${ }^{3}$ Instituto de Investigaciones Antropológicas, Facultad de Filosofía y Letras, Universidad Nacional Autónoma de México, Ciudad \\ Universitaria, 04510, México, D.F. \\ ${ }^{4}$ Escuela Nacional de Antropología e Historia, México, D.F \\ *jgama@geologia.unam.mx
}

\begin{abstract}
Resumen
El adobe como material de construcción para uso habitacional ha sido utilizado por miles de años por los pueblos indígenas de América, tanto en el suroeste de los Estados Unidos como en Mesoamérica y la región andina en Sudamérica. Actualmente el 50 por ciento de las casas del mundo están construidas con este material. La utilización del adobe representa una alternativa viable para resolver el problema de la falta de vivienda, a través de la propuesta de una casa autoconstruible de bajo costo. Sin embargo, una limitante para desarrollar tal alternativa consiste en que la mayoría de las técnicas constructivas tradicionales que utilizan materiales obtenidos a partir del suelo son resultado del conocimiento empírico. Dicho conocimiento generalmente es asistemático, varía en cada cultura y región y carece de una terminología interdisciplinaria. Por lo tanto, difícilmente esta opción ofrece, de modo directo, una base tecnológica universalmente válida.

El objetivo de este artículo consiste en contribuir al conocimiento formal relacionado con la naturaleza intrínseca y propiedades diagnósticas de uno de los materiales prehispánicos de construcción más extensamente utilizado: el adobe. Para ello, se considera viable la implementación de técnicas de medición de sus características. Con esta finalidad fueron seleccionadas y analizadas seis muestras de adobe prehispánico provenientes de los sitios arqueológicos de Zethé y Sabina Grande; ambos próximos a Huichapan, Hidalgo.

Para caracterizar formalmente a las muestras de adobe se utilizaron métodos analíticos aceptados por la Sociedad Internacional de la Ciencia del Suelo, así como por la Sociedad Americana de Pruebas y Materiales (American Society for Testing and Materials). Estos métodos incluyeron análisis físicos y químicos de rutina, complementados con análisis selectos, entre ellos: (i) determinación cuantitativa del tamaño de partícula, (ii) análisis micromorfológico, (iii) difracción por rayos X y (iv) fluorescencia de rayos X.

Las muestras analizadas se caracterizan por presentar las siguientes variables de orden cualitativo y cuantitativo: (i) domina una matriz de textura franca; (ii) muestran contenidos bajos a moderados de arcilla 1:1; (iii) predomina una densidad aparente alta; (iv) bajo coeficiente de extensión linear; (v) consistencia estable; (vi) retención de agua moderada a 33 y 1500 kPa; (vii) reacción alcalina predominante; (viii) reacción a la fenolftaleina baja a moderada; (ix) contenido pobre a moderadamente pobre de materia orgánica y de carbono total; (x) contenido bajo de $\mathrm{CaO}$ total; (xi) fracción arena dominada por vidrio y minerales volcánicos primarios; (xii) fracción arcilla dominada por cuarzo, feldespatos y haloisita; y (xiii) arreglo micromorfológico y composición mineralógica similares al de un aluvión vulcanogénico. Es posible suponer que estas características le confirieron a los adobes estudiados propiedades físicas y mecánicas de calidad aceptable como material de construcción.

Por otra parte, resulta notable que, no obstante la diferencia espacial y cronológica que existe entre los adobes investigados, las características de éstos son muy similares entre sí. Esta similitud sugiere la probabilidad de que dichos materiales fueron elaborados
\end{abstract}


bajo condiciones tecnogénicas controladas. Dicha especialización en la manufactura de adobes propone la presencia de cierto conocimiento sobre los suelos y de su transformación en elementos constructivos.

Palabras clave: adobe, arquitectura de tierra, materiales prehispánicos de construcción.

\begin{abstract}
Adobe has been used as a building material for thousands of years by indigenous peoples of America, in the southwestern United States, Mesoamerica, and Andean South America. Currently, fifty percent of the houses in the world are built with soil-derived materials. These materials may thus represent a viable solution to the problem of homelessness, by proposing a means for low-cost self-built homes. However, a limitation to developing such an alternative is that the processing techniques of building materials made from soil are the result of empirical knowledge. Such knowledge is often unsystematic, varies in each culture and region, and lacks an interdisciplinary terminology, thus precluding the recognition of universal standards for technological evaluation.

The aim of this paper is to contribute to the formal knowledge related to the intrinsic nature of one of the most widely used preHispanic construction materials: adobe. In order to do so, techniques for the quantitative measurement of the characteristics and diagnostic properties of the material were implemented. For this purpose six samples of adobe were selected and analyzed from the pre-Hispanic archaeological sites Zethé and Sabina Grande, both located near Huichapan, Hidalgo.

The samples of adobe were formally characterized by internationally accepted analytical methods used by the Society of Soil Science and by the American Society for Testing and Materials. Here, methods included routine physical and chemical analysis supplemented by selected tests, including: (i) quantitative determination of particle size, (ii) micromorphological analysis, (iii) X-ray diffraction, and (iv) X-ray fluorescence.

The samples are characterized by the following variables: (i) predominance of a loamy matrix; (ii) low to moderate content of non-expansive clay; (iii) predominance of a high bulk density; (iv) a low coefficient of linear expansion; (v) stable consistency; (vi) moderate water retention (33 and $1500 \mathrm{kPa}$ ); (vii) predominantly alkaline; (viii) low to moderate reaction to phenolphthalein; (ix) poor to moderately poor content of organic matter and total carbon; $(x)$ low content of total CaO; (xi) sand fraction dominated by primary volcanic glass and minerals; (xii) clay fraction dominated by quartz, feldspar and halloysite; and (xiii) micromorphological and mineralogical composition similar to that of a volcanogenic alluvium. We consider that these characteristics give acceptable physical and mechanical building properties to the studied adobes.

Furthermore, it is notable that the characteristics of the adobe samples are very similar to each other. This similarity suggests the likelihood that these materials were developed under controlled conditions. This specialization in adobe manufacture required a certain amount of knowledge concerning soil properties and their transformation into construction elements.
\end{abstract}

Keywords: adobe, earthen architecture, pre-Hispanic construction materials.

\section{Introducción}

El arte y la religión, así como la ciencia y la tecnología aunados a un profundo conocimiento del contexto ambiental, fueron atributos de los antiguos grupos étnicos de mesoamérica. Numerosas referencias de esas cualidades fueron documentadas en las crónicas de la conquista de México lograda por Hernán Cortés a favor del entonces Imperio español entre 1519 y 1521 . Empero, debido al cambio cultural y a la erosión de las tradiciones indígenas, mucho de ese conocimiento se ha perdido para sus aplicaciones prácticas (Gama-Castro et al., 2004, 2005).

Afortunadamente, alguna información importante, aunque fragmentada, aún perdura. Éste es el caso del conocimiento etnopedológico prehispánico (CervantesGutiérrez et al., 2005). Dicho conocimiento no solo debió ser aplicado al uso y manejo del suelo, sino también fue la base para clasificar los materiales y fracciones presentes en él. Este discernimiento determinó que estos materiales fueran utilizados para la elaboración de utensilios y la construcción de viviendas (Astrom, 1984; Daneels, 1997; Ramos-Galicia et al., 2003). Este conocimiento permitía tanto manipular los materiales edáficos, como modificar sus propiedades, es decir, transformarlos, para adecuarlos a sus necesidades.

Para lograr dicha transformación, el hombre prehispánico adicionaba a dichos materiales una serie de compuestos de origen litológico e incluso orgánico, denominados antiplásticos o desgrasantes. Con estos productos modificaba, controlaba y estabilizaba diferentes propiedades inherentes al material. Entre otras, se distinguen la plasticidad, adhesividad, compactación, espacio poroso y fundente, (Rivera-Torres y Muñoz-Díaz, 2005). Un ejemplo de esta transformación es la incorporación de materiales orgánicos (v.g. pasto) en la pasta de suelo, lo que permitía una adecuada aglutinación, alta resistencia a la intemperie 
$\mathrm{y}$, además, evitaba que los bloques una vez solidificados, tendieran a agrietarse.

De estos materiales de construcción derivados del suelo y modificados por el hombre - a los que la World Reference Base for Soil Resources (WRB, 2006) denomina como "materiales tecnogénicos"- destaca, sin duda, por sus cualidades, el adobe. Esto, fundamentalmente es debido a cinco propiedades que lo caracterizan: (i) bajo costo de fabricación y gran disponibilidad; (ii) alto ahorro de energía, haciendo uso principalmente de energías renovables; (iii) gran trabajabilidad y propiedades mecánicas óptimas en la construcción; (iv) fácil integración al ecosistema local, empleando los materiales y técnicas locales; y (v) fácil reciclamiento de los excedentes de construcción. Todas estas características conllevan un enfoque ecológico responsable con la naturaleza representando, además, una alternativa de solución al problema de vivienda mediante la propuesta de una casa autoconstruible de bajo costo.

Sin embargo, de modo paradójico, resulta sorprendente el escaso conocimiento teórico-práctico que las Ciencias de la Tierra poseen acerca de la composición, características y propiedades de dichos "materiales tecnogénicos". Probablemente, la causa principal de ello es que hasta principios de este siglo, el conocimiento etnopedológico, así como los materiales incluidos en sus definiciones carecían, para la "ciencia oficial", de credibilidad y aceptación (Barrera-Bassols y Zinck, 2003; Krasilnikov, 2002; Krasilnikov y Tabor, 2003).

Sin duda, otros factores que dificultaban la aceptación de este conocimiento empírico y sus producto eran la falta de comunicación, la heterogeneidad del conocimiento local, así como la dificultad de correlacionar el conocimiento científico con el conocimiento etnopedológico (Braimoh, 2002).

\subsection{El problema: el adobe y su terminología empírica} versus científica

La insuficiente comunicación del conocimiento empírico versus científico ha generado una limitante a la que Almeida (1998) denomina "literatura gris". Dicha limitante con frecuencia impide la creación de nuevos paradigmas que presenten soluciones a problemas específicos; en este caso, relacionados con materiales que utilizan al suelo como materia prima.

Un ejemplo lo constituye el término adobe. Así, el adobe, ha sido "definido" de varias maneras, entre las más comunes: (1) "tierra a la que se desposee minuciosamente de todo tipo de impurezas"; (2) "masa de barro" moldeada en forma de ladrillo y secada al sol"; (3) "ladrillo formado por una masa de tierra arcillosa, agua y algún aditivo, secada al sol y al aire" (Gendrop, 2001).

Como se observa, los términos tierra, masa de barro y tierra arcillosa resultan ambiguos e imprecisos para la Ciencia del Suelo. Así, en el mejor de los casos el concepto tierra puede interpretarse como la fracción inorgánica desmenuzable presente en un suelo. Sin embargo, en el caso de los conceptos masa de barro y principalmente masa de tierra arcillosa, su acepción es más compleja e incierta, generando confusiones significativas.

De acuerdo con Ericksen y Ardón (2003), esta ausencia de unificación de criterios en cuanto a la terminología ha propiciado un limitado conocimiento acerca de varias características específicas del adobe, como son: (i) composición cuantitativa de la pasta; (ii) tipo de materia orgánica adicionada; (iii) tipos de suelos o sedimentos utilizados para su elaboración; (iv) técnica constructiva; y (v) tecnología específica de elaboración en la época prehispánica. Con relación a este último inciso, se cuenta con algunas descripciones básicas en los registros del siglo XVI (Sahagún, 1956).

\subsection{Alternativa de solución}

Como alternativa de solución, se considera que, dado el origen y naturaleza intrínseca del adobe, éste es susceptible de ser analizado cualitativa y cuantitativamente mediante técnicas empleadas por la Ciencia del Suelo y la American Society for Testing and Materials (ASTM, 1985). Esto con el objetivo de formar una base cognoscitiva y en un futuro mejorar las cualidades de estos materiales como una vía de calidad en la construcción de vivienda moderna.

Para contribuir con este objetivo, fueron seleccionadas y analizadas seis muestras prehispánicas de adobe provenientes de los sitios Sabina Grande y Zethé. Ambos sitios próximos a Huichapan, Hidalgo (Figura 1). Dichas muestras fueron obtenidas durante las excavaciones realizadas entre 1989 y 1991 como parte del proyecto Valle del Mezquital (López-Aguilar, 1991, 1994; LópezAguilar et al., 1998, 2007). Las muestras estudiadas provienen de estructuras arqueológicas de tipo residencial (Sabina Grande) y ceremonial (Zethé). Actualmente no se cuenta con fechamientos que proporcionen información sobre el tiempo que los edificios fueron utilizados, pero la correlación estratigráfica con los materiales arqueológicos que se obtuvieron durante la excavación permite proponer la ocupación de Zethé para el Clásico Tardío o Epiclásico (600 d.C. -900 d.C.) y la de Sabina Grande durante el Posclásico Temprano (900 d.C. - 1200 d.C.).

Sin embargo, no existen estudios específicos sobre la utilización de adobe como elemento constructivo en la región. Su carencia impide afirmar la persistencia de una tradición constructiva desarrollada durante la época prehispánica hasta la actualidad.

\section{2. Área de estudio}

Los sitios arqueológicos de Zethé y Sabina Grande se localizan al Norte de Huichapan, el primero, y al Este, el segundo. Sus coordenadas geográficas son, respectivamente, $20^{\circ} 27^{\prime} 49^{\prime \prime} \mathrm{N}$ y $99^{\circ} 40$ '05”O y $20^{\circ} 22.44^{\prime} 47^{\prime \prime} \mathrm{N}$ y 


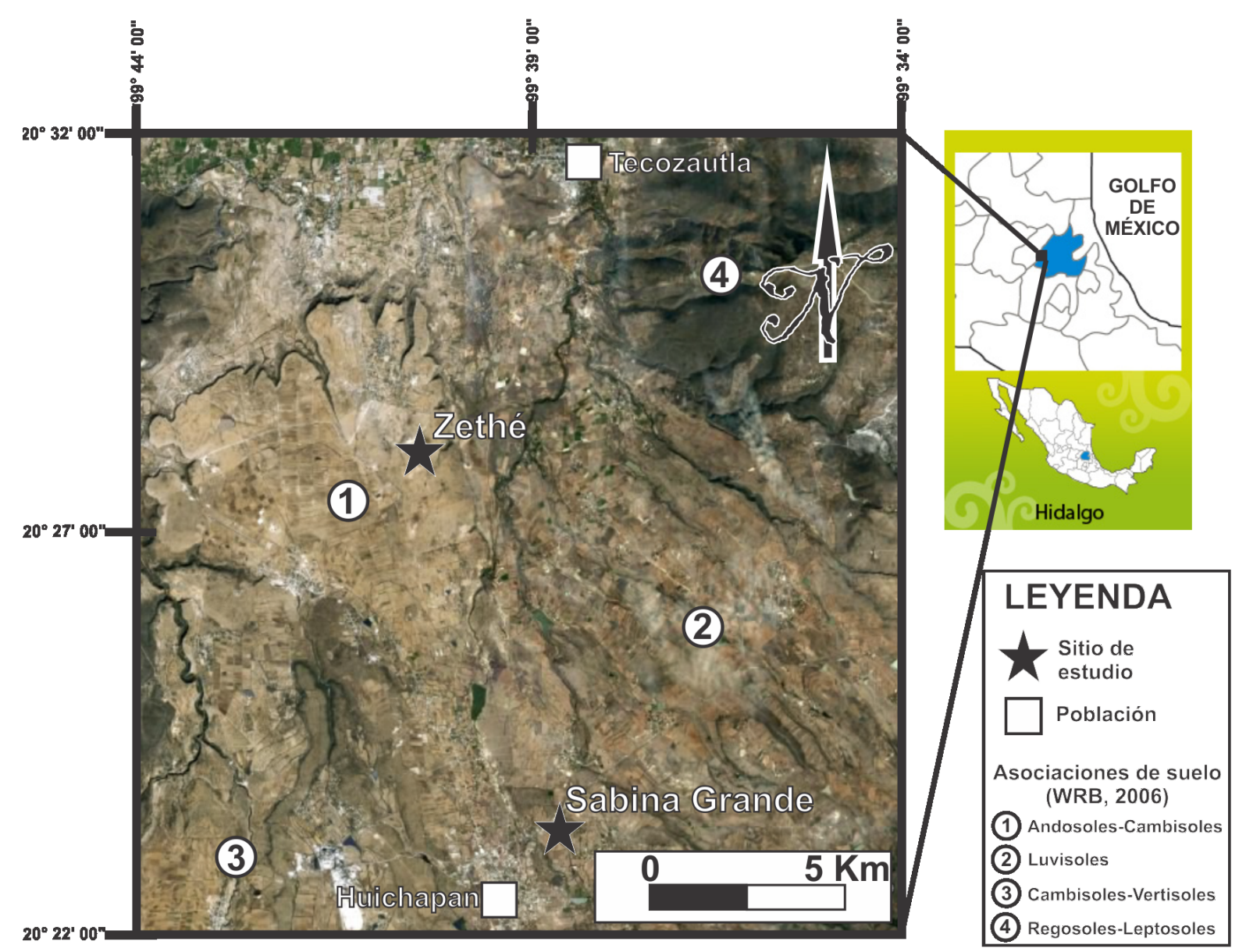

Figura 1. La imagen SPOT muestra la posición geográfica de los dos sitios de muestreo. En esta imagen también se denota la presencia de cuatro subescenas designadas, respectivamente, como 1, 2, 3 y 4 . Cada subescena está constituida por asociaciones de unidades de suelo. Zethe y Sabina Grande se ubican en una asociación de suelos volcánicos representados por Andosoles y Cambisoles (subescena 1).

99³7'52.50'O, siendo sus altitudes de $1950 \mathrm{msnm}$ y 2100 msnm (Figura 1). Con base en la interpretación de las fotografias aéreas de la región fue posible observar que la vegetación dominante en el área de estudio está formada por mezquites, huizaches, pirules, biznagas, nopales, cardones, garambullos y otras plantas típicas de climas semiáridos. El uso del suelo es predominantemente agrícola de temporal, aunque se observan pequeñas áreas sujetas a riego.

La geología de la región se compone básicamente de materiales ígneos extrusivos continentales del Terciario (Plioceno)-Cuaternario que forman parte de la Faja Volcánica Transmexicana, rodeados por materiales sedimentarios del Mioceno consistentes en depósitos clásticos de cuencas fluvio-lacustres, coluviales y de abanicos aluviales.

La imagen donde se localiza el área de estudio (Figura 1) denota que los sitios de Zethé y Sabina Grande se ubican sobre suelos de origen volcánico, probablemente Andosoles y Cambisoles con propiedades ándicas (subescena 1). Existen, además, en terrazas y pedimontes más antiguos, suelos arcillosos (subescena 2) que pueden estar representados principalmente por Luvisoles. Las planicies presentan suelos típicos de zonas áridas (subescena 3), como son Calcisoles y algunos Vertisoles, en tanto que las áreas con pendientes fuertes (subescena 4) se caracterizan por una predominancia de Leptosoles y Regosoles. Desafortunadamente, la imagen también muestra que el grado de antropización es muy alto en esta zona. Así, la deforestación es muy intensa y la erosión acelerada de tipo hídrica, es severa.

\subsection{Antecedentes arqueológicos del área de estudio}

Los sitios arqueológicos de Zethé y Sabina Grande, culturalmente se insertan dentro del complejo Xajay, o Cultura de las Mesas. La cultura Xajay, se extiende por el Noroeste del estado de Hidalgo, principalmente en los municipios de Tecozautla y Huichapan, ambos localizados en el actual valle del Mezquital, así como en el sur del estado de Querétaro, principalmente en el municipio de San Juan del Río (López-Aguilar et al., 1998). Aunque actualmente esta cultura no ha sido definida con precisión, se reconocen algunos de sus elementos representativos más característicos como son: (i) la presencia de loza cerámica rojo inciso postcocción; (ii) un patrón de asentamiento disperso; y (iii) la construcción de centros ceremoniales al borde de mesas y 
elevaciones pronunciadas (Cedeño, 1998). Asimismo, existe otro elemento importante acerca de la presencia Xajay en el norponiente del Mezquital. Dicho elemento es la presencia de un "complejo ceremonial" compuesto por cinco sitios arqueológicos, con una clara jerarquía estructural. Los sitios son, en orden de importancia jerárquica y simbólica, Pahñú, Zethé, Zidada, Taxangú y el Cerrito (López-Aguilar et al., 2007).

\subsubsection{El sitio arqueológico de Zethé}

El sitio de Zethé es un sitio ceremonial que se encuentra al oriente del complejo de los sitios antes mencionados en una meseta con un acceso hacia el sur y con varias pozas talladas en la toba volcánica. El sitio se divide en dos grandes zonas: norte y sur. La zona norte presenta tres montículos de aproximadamente 8 metros de alto y más de 9 estructuras, que forman dos conjuntos en torno a dos plazas separadas por una estructura rectangular. En la zona sur se extiende lo que se ha llamado "zona habitacional" y que consiste en por lo menos tres terrazas más y varios alineamientos que forman otras tres plazas sobre un estrato muy somero de suelo, pero que presentaba abundantes materiales arqueológicos.

Durante la primera excavación se obtuvo el registro de un piso de arcilla y de un muro de adobe. La unidad excavada incluyó un cuarto de aproximadamente $4 \times 6$ metros, con dos peanas o asientos ubicados en la pared sur; dicho espacio presentó un estrato formado por un apisonado de tiestos cerámicos bajo el cual se encontraba un piso de arcilla en buen estado de conservación, asociado a un muro de adobe. También se identificaron entre dos y tres hiladas de adobes con una altura promedio de $45 \mathrm{~cm}$ que mostraban evidencias de haber tenido un aplanado de arcilla recubriéndolos (López-Aguilar, 1994).

\subsubsection{El sitio arqueológico de Sabina Grande}

El sitio arqueológico de Sabina Grande se ubica entre las comunidades de Sabina Grande y el Cajón, al nororiente de la actual ciudad de Huichapan. Este asentamiento carece de los templos y edificios característicos de la cultura Xajay. Sin embargo, las excavaciones llevadas a cabo en 1998 descubrieron una estructura habitacional abandonada con varias ofrendas pertenecientes a dicha cultura. La unidad habitacional mostraba un muro de rocas basálticas que separaba el interior del cuarto con el exterior, el cual incluía un tiradero de tiestos rotos. El piso del cuarto estaba formado por tierra apisonada y compactada, mientras que el muro estaba vinculado a la llamada "Unidad Estratigráfica IX". Dicha Unidad fue muestreada y reportada la presencia de adobe (López-Aguilar, 1991).

\subsection{Materiales arqueológicos colectados en el área de estudio}

Es importante señalar que en la presente investigación se procuró economizar el número de muestras para análisis (seis muestras) debido a que éstas son artefactos arqueológicos escasos y que además forman parte del patrimonio cultural.

Todas las muestras de adobe analizadas se localizaron a poca profundidad, no obstante, las condiciones ambientales de intemperismo limitado favorecieron su buena preservación. También se procuró que las muestras elegidas para este estudio representaran dos sitios geográfica y temporalmente diferentes. Estas diferencias, espacial y cronológica, sirvieron para constatar la hipótesis de una especialización multitemporal en la manufactura de adobes, lo que implica la utilización de conocimientos específicos sobre los suelos y su transformación en elementos constructivos.

\section{Materiales y métodos}

Las muestras fueron analizadas en los laboratorios del Instituto de Geología, UNAM. Las muestras colectadas en Zethé son referidas en este estudio como M1, M2, M3 y M4. Las muestras M5 y M6 son representativas de Sabina Grande. En estas seis muestras se realizaron tanto análisis de rutina como análisis selectos, los cuales son descritos a continuación.

\subsection{Análisis de rutina}

Dichos análisis comprendieron determinaciones físicas y químicas de las muestras. Los análisis físicos realizados fueron los siguientes: (a) color. Se determinó el color que caracteriza a los adobes, en seco y húmedo. Este análisis se realizó con base en las tablas de color Munsell (1975); (b) textura. Se realizó de acuerdo con el método de Bouyoucos (1963); (c) cuantificación de la densidad aparente. Fue estimada de acuerdo con la metodología recomendada por el Soil Conservation Service (SCS, 1984); (d) determinación del coeficiente de extensión lineal (COLE). Se realizó con base en los procedimientos utilizados por la American Society for Testing and Materials (ASTM, 1985); (e) determinación de la consistencia en húmedo y seco. Fue evaluada siguiendo los lineamientos propuestos por Schoeneberger et al. (1998); y (f) retención de agua a 33 y $1500 \mathrm{kPa}$. Fue cuantificada con base en el Soil Conservation Service (SCS, 1984).

Los análisis químicos incluyeron las siguientes determinaciones: (a) reacción al $\mathrm{HCl}(10 \%)$. Fue evaluada con base en la metodología utilizada por el Soil Conservation Service (SCS, 1984); (b) reacción a la fenolftaleina. Fue estimada de acuerdo con con el método propuesto por la Organización de las Naciones Unidas para la Agricultura y la Alimentación (FAO), el Centro Internacional de Referencia e Información en Suelos y la Sociedad Internacional de las Ciencias del Suelo (FAO et al., 1998); (c) pH en agua relación 1:2.5. Fue determinado con base en la metodología del Soil Conservation Service (SCS, 1984); (d) pH en NaF $1 \mathrm{~N}$, relación 1:50. Fue cuantificado en un potenciómetro 
Hanna, modelo HI 9025, siguiendo la metodología propuesta por el Soil Conservation Service (1984); y (e) materia orgánica y (f) carbono total, fueron determinados de acuerdo al Natural Resources Conservation Service (NCRS, 2004).

\subsection{Análisis selectos}

Dichos análisis incluyeron las siguientes determinaciones:

Análisis granulométrico. Efectuado con base en la metodología propuesta por el Soil Conservation Service (SCS, 1984), utilizando el siguiente procedimiento metodológico. Separación y cuantificación de las partículas de arena, limo y arcilla de cada una de las muestras de adobe. La cuantificación de las partículas minerales de tamaño de arenas fue realizada utilizando diferentes tamices. Las fracciones media (arenas muy finas y limo) y fina (arcilla) se estimaron por sedimentación de las partículas a través del método de la pipeta. El método de pipeta utilizado requirió tratar la muestras con diferentes reactivos para eliminar los agentes cementantes y asegurar una máxima dispersión de las partículas (Gee y Or, 2002).

Análisis micromorfológico. Las secciones delgadas fueron preparadas a partir de muestras inalteradas impregnadas con resina epóxica (con índice de refracción 1.64) y analizadas bajo microscopio petrográfico. Las descripciones e interpretaciones se realizaron con base en la terminología y metodología de Bullock et al. (1985) y Stoops (2003).

Difracción de rayos X. Los difractogramas se obtuvieron en un difractómetro Shimadzu XRD-6000 equipado con filtro de $\mathrm{Ni}$, tubo de cobre y monocromador. Las seis muestras de adobe se analizaron como roca total en fracción no orientada. El procedimiento utilizado se describe brevemente a continuación.

Antes de la medición se procedió a la eliminación de materia orgánica con peróxido de oxígeno según la metodología propuesta por el Soil Conservation Service (SCS, 1984). Posteriormente, las muestras se molturaron y homogenizaron mediante un mortero de ágata y se analizaron utilizando un portamuestras de aluminio en el intervalo angular $2 \theta$ de $4^{\circ}$ a $70^{\circ}$ con escaneo continuo y escaneo por pasos para determinar su mineralogía. Paralelamente y para determinar la mineralogía de la fracción arcilla, en todas las muestras se separó la fracción $<2$ micras y se prepararon y analizaron por difracción de rayos $\mathrm{X}$ los agregados orientados.

Fluorescencia de Rayos X. Para este análisis fue seleccionada la muestra M4, debido a que se estimó como representativa del material estudiado. La química de la muestra M4 se analizó con un espectrómetro secuencial de fluorescencia de rayos X del Instituto de Geología, modelo Siemens SRS 3000, equipado con tubo de rodio y ventana de berilio. Los resultados se expresan como porcentaje de óxidos.

\section{Resultados y Discusión}

\subsection{Análisis de rutina}

Los análisis de rutina realizados a las muestras de adobe se encuentran sintetizados en las Tablas 1 y 2 . Como puede observarse, la Tabla 1 presenta las principales características físicas y mecánicas que distinguen a las muestras. La Tabla 2 contiene varias de las características químicas más relevantes de estos materiales.

Características físicas: (a) Color. Con respecto al color en seco y húmedo que caracteriza a las muestras, éste oscila de pardo a pardo brillante y pardo oscuro. Dichos colores están condicionados principalmente por los porcentajes de materia orgánica presente en las muestras. Los colores más oscuros corresponden con las muestras que presentan el mayor contenido de materia orgánica (M1, M2 y M6) y de carbón total (Tabla 2); (b) Textura. En todos los casos, la textura de los adobes es característica de un migajón

Tabla 1. Características físicas y mecánicas de los adobes estudiados. Color: $\mathrm{S}=$ color en seco, $\mathrm{H}=$ color en húmedo (Munsell, 1975); Textura: $\mathrm{A}=$ porcentaje de arena; $\mathrm{L}=$ porcentaje de limo; $\mathrm{C}=$ porcentaje de arcilla; D.A.: = densidad aparente; COLE: $=$ coeficiente de extensión lineal; Consistencia: $\mathrm{S}=$ en seco, $\mathrm{H}=$ en húmedo.

\begin{tabular}{|c|c|c|c|c|c|c|}
\hline Muestra & Color & $\begin{array}{c}\text { Textura } \\
(\%) \\
\end{array}$ & $\begin{array}{r}\text { D. A } \\
\left(\mathrm{g} / \mathrm{cm}^{3}\right) \\
\end{array}$ & COLE & Consistencia & $\begin{array}{c}\text { Retención } \\
\text { de Agua } \\
(\%) \\
\end{array}$ \\
\hline M1 & $\begin{array}{l}\text { S } 10 \text { YR } 4 / 2 \\
\text { H10YR } 3 / 2\end{array}$ & $\begin{array}{l}\text { A } 52.74 \\
\text { L } 32.86 \\
\text { C } 14.38 \\
\text { Franco }\end{array}$ & 1.39 & $\begin{array}{c}0.031 \\
3 \%\end{array}$ & $\begin{array}{c}\mathrm{S} \\
\text { Ligeramente dura } \\
\mathrm{H} \\
\text { Moderadamente } \\
\text { friable }\end{array}$ & $\begin{array}{c}33 \mathrm{kPa} \\
28.84 \\
\\
1500 \mathrm{kPa} \\
12.19\end{array}$ \\
\hline M2 & $\begin{array}{l}\text { S } 10 \text { YR } 4 / 2 \\
\text { H } 10 \text { YR } 3 / 2\end{array}$ & $\begin{array}{l}\text { A } 52.45 \\
\text { L } 34.10 \\
\text { C } 13.42 \\
\text { Franco }\end{array}$ & 1.38 & $\begin{array}{c}0.02 \\
2 \%\end{array}$ & $\begin{array}{c}\mathrm{S} \\
\text { Ligeramente dura } \\
\mathrm{H} \\
\text { Moderadamente } \\
\text { friable }\end{array}$ & $\begin{array}{c}33 \mathrm{kPa} \\
29.3 \\
1500 \mathrm{kPa} \\
12.93\end{array}$ \\
\hline M3 & $\begin{array}{l}\text { S } 7.5 \text { YR } 5 / 4 \\
\text { H } 7.5 \text { YR } 4 / 2\end{array}$ & $\begin{array}{l}\text { A } 39.90 \\
\text { L } 44.39 \\
\text { C } 16.68 \\
\text { Franco }\end{array}$ & 1.4 & $\begin{array}{c}0.036 \\
3.50 \%\end{array}$ & $\begin{array}{c}\mathrm{S} \\
\text { Dura } \\
\mathrm{H} \\
\begin{array}{c}\text { Moderadamente } \\
\text { friable }\end{array}\end{array}$ & $\begin{array}{c}33 \mathrm{kPa} \\
28.29 \\
1500 \mathrm{kPa} \\
10.63\end{array}$ \\
\hline M4 & $\begin{array}{l}\text { S } 7.5 \text { YR } 5 / 6 \\
\text { H } 7.5 \text { YR } 4 / 2\end{array}$ & $\begin{array}{l}\text { A } 35.55 \\
\text { L } 44.27 \\
\text { C } 20.21 \\
\text { Franco }\end{array}$ & 1.43 & $\begin{array}{c}0.052 \\
5 \%\end{array}$ & $\begin{array}{c}\mathrm{S} \\
\text { Dura } \\
\mathrm{H} \\
\text { Moderadamente } \\
\text { friable a firme }\end{array}$ & $\begin{array}{c}33 \mathrm{kPa} \\
20.56 \\
1500 \mathrm{kPa} \\
13.43\end{array}$ \\
\hline M5 & $\begin{array}{l}\text { S } 7.5 \text { YR } 5 / 6 \\
\text { H } 7.5 \text { YR } 4 / 2\end{array}$ & $\begin{array}{l}\text { A } 31.83 \\
\text { L } 45.50 \\
\text { C } 22.70 \\
\text { Franco }\end{array}$ & 1.45 & $\begin{array}{c}0.058 \\
5.50 \%\end{array}$ & $\begin{array}{c}\text { S } \\
\text { Muy dura } \\
\mathrm{H} \\
\text { Moderadamente } \\
\text { friable a firme }\end{array}$ & $\begin{array}{c}33 \mathrm{kPa} \\
28.25 \\
\\
1500 \mathrm{kPa} \\
14.05\end{array}$ \\
\hline M6 & $\begin{array}{l}\text { S } 10 \text { YR } 4 / 2 \\
\text { H } 10 \text { YR } 3 / 2\end{array}$ & $\begin{array}{l}\text { A } 47.57 \\
\text { L } 31.35 \\
\text { C } 21.11 \\
\text { Franco }\end{array}$ & 1.45 & $\begin{array}{c}0.052 \\
5 \%\end{array}$ & $\begin{array}{c}\text { S } \\
\text { Dura } \\
\mathrm{H} \\
\text { Moderadamente } \\
\text { friable a firme }\end{array}$ & $\begin{array}{c}33 \mathrm{kPa} \\
26.89 \\
\\
1500 \mathrm{kPa} \\
11.38\end{array}$ \\
\hline
\end{tabular}


Tabla 2. Características químicas de los adobes estudiados

\begin{tabular}{|c|c|c|c|c|c|c|}
\hline Muestra & $\begin{array}{c}\text { Reacción } \\
\text { al HCl } \\
(10 \%) \\
\end{array}$ & $\begin{array}{c}\text { Reacción a la } \\
\text { fenolftaleína } \\
(1 \%) \\
\end{array}$ & $\begin{array}{c}\text { pH en } \\
\text { NaF 1N } \\
\text { Rel. 1:50 } \\
\end{array}$ & $\begin{array}{c}\text { pH en } \\
\text { agua } \\
\text { Rel. } 1: 2.5 \\
\end{array}$ & $\begin{array}{c}\text { Materia } \\
\text { orgánica } \\
(\%) \\
\end{array}$ & $\begin{array}{c}\text { Carbono } \\
\text { total } \\
(\%) \\
\end{array}$ \\
\hline M1 & Nula & ligera & 8.73 & 7.87 & 1.29 & 0.65 \\
\hline M2 & Nula & moderada & 9.04 & 8.24 & 1.26 & 0.63 \\
\hline M3 & Nula & ligera & 7.8 & 6.56 & 0.97 & 0.49 \\
\hline M4 & Nula & fuerte & 9.09 & 8.87 & 0.79 & 0.39 \\
\hline M5 & Nula & fuerte & 8.84 & 8.7 & 0.54 & 0.27 \\
\hline M6 & Nula & fuerte & 8.51 & 8.55 & 1.53 & 0.77 \\
\hline
\end{tabular}

franco, donde la relación limo-arcilla, siempre es mayor de 1. Esto indica la probabilidad de una edad geológica joven del material utilizado para construir los adobes, así como de un estatus de intemperismo poco agresivo; (c) Densidad aparente (D.A.). Es obvio que los valores altos de D.A. que caracterizan a todos los adobes resultan de los procesos tecnogénicos y factores involucrados (v.g. hidroconsolidación por secado, compactación) en la elaboración de estos materiales; (d) COLE. Los valores que caracterizan el coeficiente de extensión lineal de las muestras son muy bajos y están estrechamente relacionados con los porcentajes bajos y tipo de arcilla presentes. En este caso, con arcillas 1:1 no expansivas del grupo del caolín, como es la haloisita. Dichas características resultan ideales cuando se procura evitar el riesgo y deterioro que propician en obras habitacionales la presencia de materiales con índices altos de expansión-contracción (v.g. arcilla 2:1 del grupo de las bentonitas); (e) Consistencia en húmedo y seco. La consistencia en seco de los adobes analizados varía de ligeramente de dura a muy dura, presentando una consistencia en húmedo que oscila de moderadamente friable a firme. Es probable que los valores originales relativos a esta característica hayan decrecido gradualmente a través del tiempo, principalmente por la acción del intemperismo. Sin embargo, este material aún presenta valores aceptables de estabilidad y baja erodabilidad; y (f) porcentaje de retención de agua. Se considera que todos los adobes presentan un valor moderadamente alto, el cual resulta, en gran parte, de los contenidos de materiales muy porosos e hidrófilos en su matriz, como es la pómez parcialmente intemperizada (Gama-Castro et al., 2000).

Características químicas: (a) Reacción al $\mathrm{HCl}(10 \%)$. En ninguna de las muestras de adobe hubo reacción al $\mathrm{HCl}$. Esto resulta congruente con los contenidos de $\mathrm{Ca}^{++}$ total que se detectaron en los análisis; y (b) Reacción a la fenolftaleina. La reacción moderada a fuerte que presentan algunas muestras (M2, M4, M5 y M6) al reactivo es atribuible al nivel de alcalinidad que presentan ( $\mathrm{pH}$ en agua > 7.7) y no a la existencia de minerales amorfos como el alófano o la ferrihidrita. El origen de dicha alcalinidad aún no ha sido establecido, aunque existe la hipótesis de que está relacionado con la formación de algunas zeolitas presentes en la fracción arena fina de las muestras.

Con respecto a los valores obtenidos para (d) $\mathrm{pH}$ en $\mathrm{NaF}$ $1 \mathrm{~N}$, todos son $<10$. Estos valores corroboran la ausencia de minerales amorfos. Dicho déficit en la presencia de minerales amorfos sugiere una juventud evolutiva y un escaso desarrollo pedogenético alcanzado por los suelos que fueron utilizados para la elaboración de los adobes. Este déficit de minerales amorfos es muy común en los suelos clasificados como Andosoles vítricos o Regosoles téfricos (Gama-Castro et al., 2000). Ambos suelos son frecuentes en la zona de estudio.

Finalmente, con respecto a los contenidos que muestran los adobes en relación con (e) materia orgánica y (f) carbono total son, en general, pobres (M3, M4, M5 y M6) a moderadamente pobres (M1 y M2). Esto se debe en gran parte a que las técnicas analíticas empleadas para su determinación sólo son sensibles para evaluar los contenidos de materia orgánica que ha sido oxidada. En este caso, los materiales de origen orgánico, por estar incluidos en la matriz de adobe y aislados del medio ambiente, se han oxidado en cantidad escasa y lentamente.

\subsection{Análisis selectos}

Análisis granulométrico. La Tabla 3 muestra la distribución del tamaño de las partículas que conforman la matriz de los adobes estudiados. Destacan en esta tabla los siguientes aspectos: (1) la clase textural de migajón franco domina en las seis muestras de adobe analizadas; (2) se observa que los tamaños de arenas finas, muy finas y limo son los constituyentes predominantes en la matriz de dichas muestras; (3) los porcentajes de arcilla varían de bajos (M1, M2) a moderados (M3, M4, M5 y M6), siendo que la mayor acumulación de arcilla apenas rebasa el $20 \%$; y (4) como se observa en la Figura 2, la distribución y porcentajes de los diversos tamaños de partícula resultan muy similares en todas las muestras. Con base en estas características de tamaño de partícula se puede inferir lo siguiente:

1. Un material con textura de migajón franco resulta ideal, desde un punto de vista artesanal, por ser fácil de amasar y moldear para diferentes usos. Esto debido a que no presenta una consistencia muy dura en seco, siendo en húmedo moderadamente friable a firme, lo que permite estabilidad estructural (Tabla 1). Además, cuando un material con estas características texturales se expone al sol, pierde gradualmente el agua retenida sin deformar su volumen notablemente (COLE, Tabla 1). Esta pérdida de agua (desecación) y, consecuentemente, de espacio poroso, genera su paulatina consolidación (hidroconsolidación) y su posterior compactación. De modo análogo, parte de esta dinámica se presenta en la naturaleza contribuyendo a la formación de algunos tipos de tepetates (Solleiro et al., 2003; Gama-Castro et al., 2007).

2. Por otra parte, el hecho de que las fracciones de arenas finas, muy finas y limos sean dominantes (Tabla 
Tabla 3. Análisis del tamaño de partículas.

\begin{tabular}{cccccccc}
\hline \multirow{2}{*}{ Tamaño de partícula (mm) } & $\begin{array}{c}\mathbf{2 . 0}-\mathbf{1 . 0} \\
\mathbf{( \% )}\end{array}$ & $\begin{array}{c}\mathbf{1 . 0}-\mathbf{0 . 5} \\
\mathbf{( \% )}\end{array}$ & $\begin{array}{c}\mathbf{0 . 5}-\mathbf{0 . 2 5} \\
\mathbf{( \% )}\end{array}$ & $\begin{array}{c}\mathbf{0 . 2 5}-\mathbf{0 . 1 0} \\
\mathbf{( \% )}\end{array}$ & $\begin{array}{c}\mathbf{0 . 1 0 - 0 . 0 5} \\
\mathbf{( \% )}\end{array}$ & $\begin{array}{c}\mathbf{0 . 0 5 - 0 . 0 0 2} \\
\mathbf{( \% )}\end{array}$ & $\begin{array}{c}<\mathbf{0 . 0 0 2} \\
\mathbf{( \% )}\end{array}$ \\
\hline M1 & 1.58 & 4.97 & 11.12 & 25.22 & 9.85 & 32.86 & 14.38 \\
M2 & 1.54 & 3.77 & 10.9 & 24.61 & 11.63 & 34.1 & 13.42 \\
M3 & 0.4 & 2.1 & 6.45 & 18.44 & 11.51 & 44.39 & 16.68 \\
M4 & 0.48 & 1.24 & 3.93 & 18.52 & 11.38 & 44.27 & 20.21 \\
M5 & 0.24 & 1.24 & 4.62 & 15.49 & 10.24 & 45.5 & 22.7 \\
M6 & 3.04 & 6.03 & 10.99 & 19.03 & 8.48 & 31.35 & 21.11 \\
\hline
\end{tabular}

$2.0-1.0 \mathrm{~mm}=$ arena muy gruesa; $1.0-0.5 \mathrm{~mm}=$ arena gruesa; $0.5-0.25 \mathrm{~mm}=$ arena media; $0.25-0.10 \mathrm{~mm}=$ arena fina; $0.10-0.05 \mathrm{~mm}=$ arena muy fina; $0.05-0.002 \mathrm{~mm}=$ limo; $<0.002 \mathrm{~mm}=$ arcilla.

Tamaño dep articulas

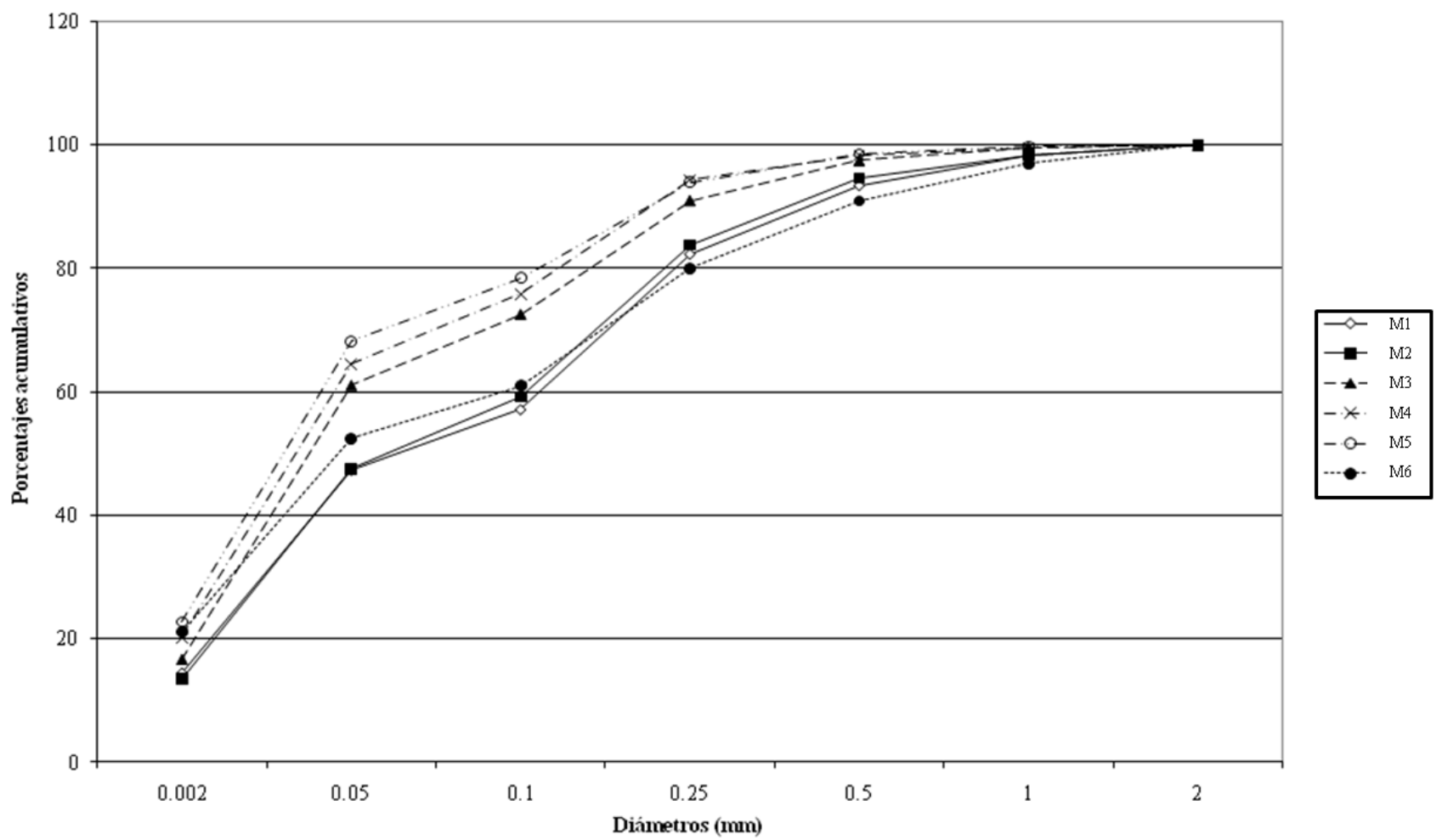

Figura 2. La gráfica muestra que la distribución de los diversos tamaños de partícula resulta muy similar en todas las muestras de adobe. Las arenas finas $(0.25-0.10 \mathrm{~mm})$, arenas muy finas $(0.10-0.05 \mathrm{~mm})$ y limo $(0.05-0.002 \mathrm{~mm})$ son predominantes en la matriz de dichas muestras. Estos tipos texturales se consideran análogos a los que caracterizan a varios suelos volcánicos que muestran un desarrollo pedológico ligero a moderado.

3), aunado a una pobreza en materia orgánica (Tabla 2), confiere a estos materiales una erodabilidad alta y un riesgo potencial a ser erosionados por el agua, particularmente en zonas lluviosas. Desde luego que el grado de consolidación y compactación que presentan los adobes atenúa este riesgo, aunque solamente durante algún tiempo. Esto implica que las construcciones de adobe requieren un mantenimiento periódico.

3. Finalmente, la similitud que presentan estos materiales en su distribución y tamaño de partículas (Figura 2), sugiere que hubo una cuidadosa selección de la materia prima para la elaboración de los adobes así como un control en su producción.

Análisis micromorfológico. La Figura 3 muestra en seis imágenes una síntesis de los rasgos micromorfológicos más representativos de las muestras estudiadas. Atendiendo a dichos rasgos fue evidente la presencia de una microestructura granular (Figura 3a). Dicha estructura presenta un desarrollo que oscila de débil a moderado. En algunas zonas, esta estructura llega a compactarse para conformar bloques angulares pobremente desarrollados (separados entre sí por poros planares poco desarrollados). Otro proceso muy 

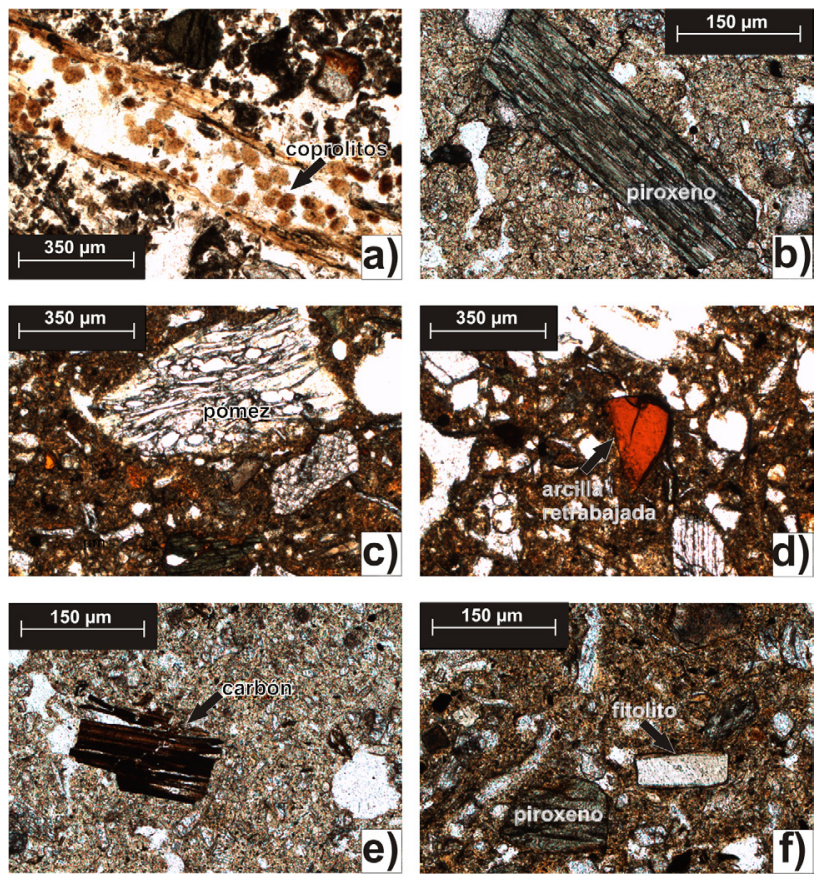

Figira 3. La imagen integra los rasgos micromorfológicos diagnósticos más comunes para las seis muestras de adobe analizadas. Las imágenes $\mathrm{a}, \mathrm{b}$ y f corresponden con muestras de los adobes muestreados en Zethé. Las imágenes c, d, y e pertenecen a los adobes obtenidos en el sitio de Sabina Grande.

distintivo en esta muestra es la bioturbación, evidenciada por la presencia de huellas de raíces, cámaras y rasgos excretales. Dichos rasgos llegan a presentar coalescencia, incorporándose a la estructura microgranular.

Con respecto a los principales componentes minerales presentes en la fracción arena de las muestras colectadas en Zethé, éstas exhiben el dominio de un ensamble volcanogénico, constituido por vidrio volcánico (ubicado en esquirlas), plagioclasas (de composición intermedia a básica, principalmente andesina), anfíboles, piroxenos (Figura 3b) y fragmentos de roca (de hasta $2 \mathrm{~mm}$ de largo).

En las muestras provenientes de Sabina Grande, el vidrio volcánico es menos dominante, cediéndole lugar a la plagioclasa intermedia (Figura 3c). En estas muestras los fragmentos de roca están compuestos principalmente por pómez (con estructura fluidal y vesicular) así como litoclastos con textura porfídica (con parte de matriz vítrea que circunda a los microlitos de plagioclasa intermedia). Los rasgos de intemperismo no son comunes salvo algunas alteraciones de "dientes de sierra" y de "texturas de tamiz" presentes en piroxenos de tamaño de arena fina. En lo que corresponde a los fragmentos de roca, algunos de sus componentes ferromagnesianos denotan alteraciones a óxidos de Fe, ubicados en "halos" alrededor de los fenocristales.

También se observó que existen componentes particularmente interesantes dentro de la fracción arena: óxidos de Fe (goethita, observable bajo luz transmitida) y fragmentos de materiales arcillosos retrabajados (notables debido a su continuidad óptica; Figura 3d). La fracción arena fina y limo también está dominada por los componentes volcanogénicos, y dicho tamaño de partícula fue dominante en las muestras de Sabina Grande.

Dentro de los componentes orgánicos gruesos, observados en las láminas (Figura 3e), resalta la presencia de fragmentos de carbón, aunque éstos no son muy abundantes $(<3 \%)$. En general, los componentes orgánicos presentan un estado de degradación de moderado a avanzado (identificable por su tendencia a la isotropía). En todos los materiales estudiados fueron identificados fitolitos (Figura 3f).

Se observó en las muestras de Zethé que respecto al material fino inferior a $2 \mu \mathrm{m}$ es difícil de establecer su identificación como mineral arcilloso bien cristalizado. Esto debido a su probable enmascaramiento por pigmentación de la materia orgánica. Sin embargo, en algunas zonas fue posible observar la presencia de arcilla limpia (bajo luz polarizada plana) con tonalidades amarillo-rojizas, que sugieren cierta impregnación con óxidos de $\mathrm{Fe}$, con colores de interferencia bajos y continuos (denotando la probable presencia de minerales arcillosos de estructura 1:1). Sin embargo, dichas áreas son muy localizadas y se presentan alrededor de minerales tamaño arena gruesa o fragmentos de roca (resaltando zonas de tensión a manera de estrías), que en ocasiones son rellenados en sitios porosos.

En las muestras procedentes de Sabina Grande el material fino es prácticamente indiferenciado, no se observan regiones con continuidad óptica, lo que sugiere el dominio de materiales de baja cristalinidad.

Difracción de rayos X. Para las seis muestras estudiadas se obtuvieron difractogramas con resolución media debido a la presencia de ópalo, vidrio y arcillas de baja a muy baja cristalinidad (Figura 4). No obstante, fue posible obtener los siguientes resultados:

1. Todas las muestras presentan las mismas fases mineralógicas y únicamente se diferencian ligeramente entre sí por las abundancias relativas de las mismas (Tabla 4). En todas las muestras se ha podido identificar la presencia de cuarzo, haloisita $7.2 \AA$, feldespato tipo plagioclasa y ópalo como minerales principales. En casi todas se ha podido observar la presencia de anfíbol como fase accesoria y pequeñas cantidades de hematita. Las fases accesorias nunca se presentan en proporción superior al $5 \%$.

2. Los principales minerales detectados en las muestras de adobe son:

a. Cuarzo. Se identifica claramente por la presencia de picos muy bien definidos con distancia interplanar de 3.34 Å y $4.26 \AA ;$

b. Haloisita. Se caracteriza por presentar picos anchos de difracción con distancias interplanares de $7.2 \AA(001), 4.4 \AA$ (02), $2.56 \AA$ (20) y 1.48-1.49 $\AA$ (060). El pico 060 es bastante ancho, indicando la presencia de un mineral dioctaédrico. $\mathrm{La}$ reflexión no basal de $4.4 \AA$ es mayor que la reflexión basal $7.2 \AA$, lo que se puede asociar con la morfología no planar de dicho mineral. La baja cristalinidad del mineral también 


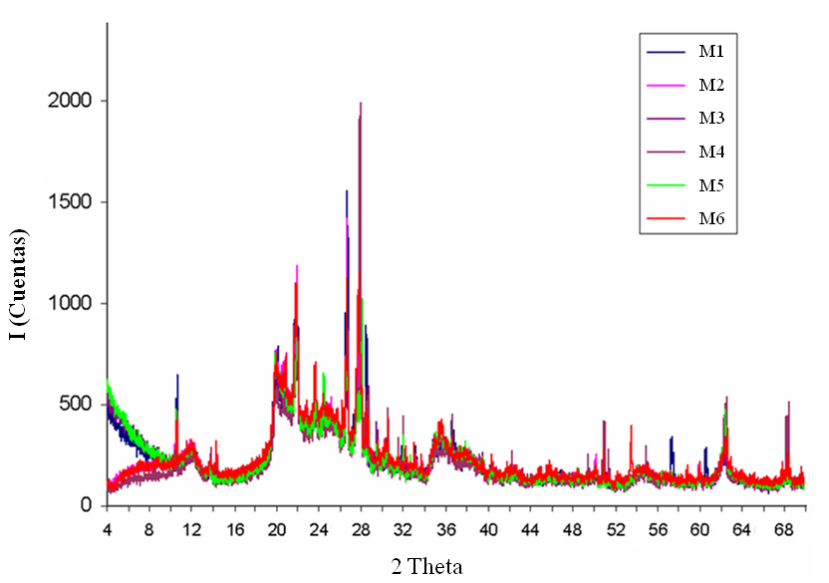

Figura 4. El difractograma exhibe las mismas fases mineralógicas en las seis muestras de adobe analizadas. Estas fases se diferencian ligeramente entre sí por las abundancias relativas de los minerales presentes.

Tabla 4. Composición mineralógica de los adobes estudiados. El orden con el que se citan los minerales en la Tabla 4 está en función de su abundancia relativa. Se citan en orden decreciente.

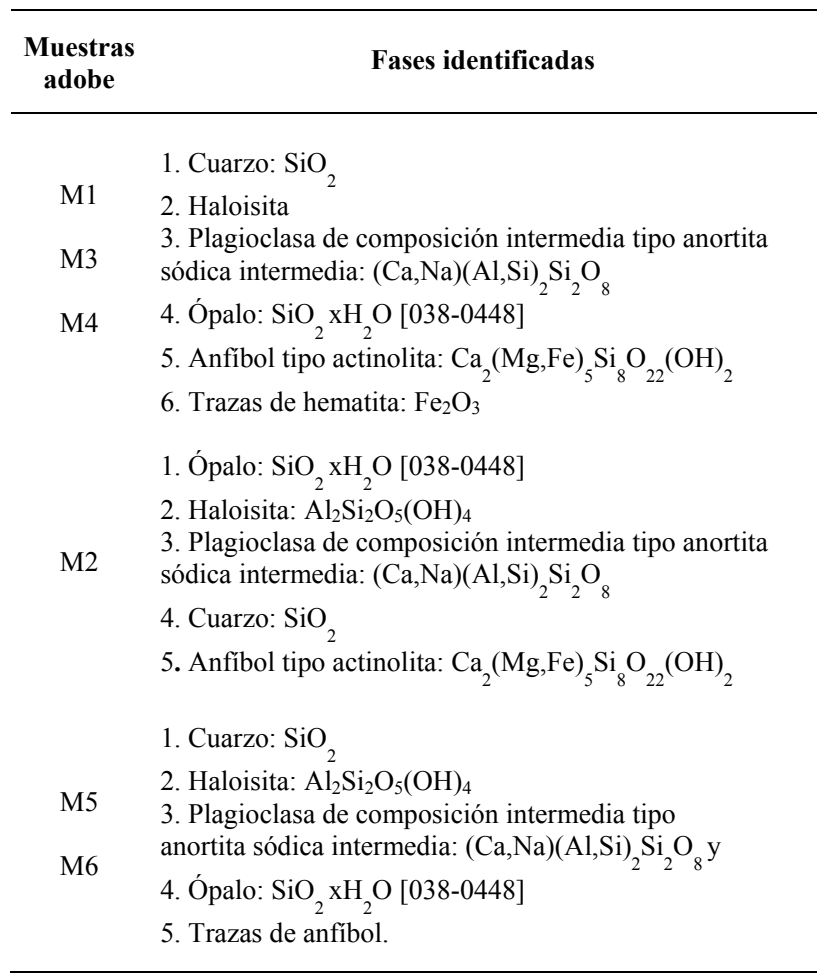

se puede asociar a la presencia de cristales de tamaño muy pequeño. Todo ello y su desaparición por calentamiento de la muestra a $550{ }^{\circ} \mathrm{C}$ confirma que el mineral en cuestión es haloisita $7 \AA$;

c. Sílice poco cristalina. En los difractogramas de muestras de adobe se identificaron siempre los siguientes picos de cristobalita (low cristobalite): $\mathrm{d}_{101}$ a 4.05-4.07 $\AA \mathrm{y}$ $\mathrm{d}_{200}$ a $2.50-2.51 \AA$ y en algunos casos los picos $\mathrm{d}_{110}$ a 3.53 $\AA, \mathrm{d}_{111}$ a $3.13 \AA \mathrm{y} \mathrm{d}_{102}$ a $2.84 \AA$. También se encontraron los siguientes picos de tridimita (low tridymite): $\mathrm{d}_{040}$ a 4.33 $\AA, \mathrm{d}_{211}$ a $4.11 \AA, \mathrm{d}_{211}$ a $3.87 \AA \mathrm{y} \mathrm{d}_{042}$ a $2.97 \AA$ en las seis muestras estudiadas.

De acuerdo con la clasificación sedimentaria de los ópalos se puede considerar que el ópalo que se presenta en las muestras de adobe son de tipo CT con predominio de picos de cristobalita y en menor proporción de tridimita; y

d. Feldespatos. Se han podido identificar como plagioclasas de composición intermedia por la presencia de dos reflexiones importantes entre $3.22 \AA$ y $3.18 \AA$ y una reflexión de menor tamaño entre $6.3 \AA$ y $6.4 \AA$.

En general, los minerales detectados por difracción de rayos $\mathrm{X}$ en este estudio son, en México, comunes de encontrar en suelos de origen volcánico, jóvenes y poco desarrollados. Sin embargo, con frecuencia dichos minerales también se presentan en los aluviones vulcanogénicos, en los que predomina en su matriz, más de 65 por ciento de vidrio volcánico y materiales piroclásticos retrabajados, ambos mezclados con sedimentos, fragmentos líticos y materia orgánica provenientes de otras fuentes (GamaCastro et al., 2000).

Fluorescencia de rayos X. Como se observa en la Tabla 5, la composición química total de la muestra M4 es intermedia. En dicha muestra se infiere que los elementos alcalinos, la sílice y el aluminio, son constituyentes importantes de las plagioclasas, vidrio volcánico y en menor proporción de las zeolitas silícicas y arcillas de tipo haloisita. También se infiere lo siguiente:

1. El hierro se debe relacionar con la presencia de anfíbol $\mathrm{y}$ hematita. El contenido en $\mathrm{Mg}^{++}$es bastante menor que el de hierro y se debe asociar únicamente con el anfíbol;

2. No se conoce en qué minerales puede encontrarse el Ti ni el Ba, pero no se descarta que se encuentren también en el vidrio;

3. Con respecto al $\mathrm{CaO}$, se debe mencionar que, aunque sus porcentajes son moderadamente bajos (Tabla 5), su presencia es importante, ya que coadyuva como estabilizante de los adobes para el mejoramiento de su resistencia mecánica; y

4. Se estima que la pérdida de volátiles por calcinación (PXC) en todas las muestras estudiadas es muy alta ( $>10 \%$ ), y eso se relaciona con la presencia de arcillas, zeolitas y vidrio volcánico probablemente hidratado.

\section{Conclusiones}

La diferencia espacial y cronológica que existe entre los sitios de Zethé y Sabina Grande permite constatar la hipótesis de una especialización multitemporal en la manufactura de adobes. Esto conlleva a inferir que la población de estos sitios poseía conocimientos específicos sobre los suelos y su transformación en elementos constructivos.

Como muestran los resultados de los análisis físicos, químicos y mineralógicos, se infiere que los habitantes prehispánicos de Zethé y Sabina Grande utilizaron materiales 
Tabla 5. Análisis por fluorescencia de Rayos X

\begin{tabular}{|c|c|c|c|c|c|c|c|c|c|c|}
\hline $\begin{array}{l}\mathrm{SiO}_{2} \\
(\%)\end{array}$ & $\begin{array}{c}\mathbf{A l}_{2} \mathbf{O}_{3} \\
(\%)\end{array}$ & $\begin{array}{r}\mathrm{CaO} \\
(\%)\end{array}$ & $\begin{array}{c}\mathrm{Na}_{2} \mathrm{O} \\
(\%)\end{array}$ & $\begin{array}{l}\mathbf{K}_{2} \mathbf{O} \\
(\%)\end{array}$ & $\begin{array}{l}\mathrm{TiO}_{2} \\
(\%)\end{array}$ & $\begin{array}{c}\mathrm{Fe}_{2} \mathrm{O}_{3} \\
(\%)\end{array}$ & $\begin{array}{c}\text { MgO } \\
(\%)\end{array}$ & $\begin{array}{r}\mathrm{BaO} \\
(\%)\end{array}$ & $\begin{array}{r}\text { PXC* } \\
(\%)\end{array}$ & $\begin{array}{c}\text { TOTAL } \\
(\%)\end{array}$ \\
\hline 58.6 & 16.5 & 1.98 & 1.27 & 2.01 & 1.36 & 5.73 & 0.96 & 0.44 & 11.1 & 98.7 \\
\hline
\end{tabular}

$* \mathrm{PXC}=$ perdida por calcinación

pedológicos provenientes de su entorno inmediato.

Los datos analíticos obtenidos indican que la materia prima con que fueron construidos los adobes es análoga a la que presentan en su complejo de alteración los suelos volcánicos poco evolucionados (v.g. Regosol téfrico, Andosol vítrico) e incluso a la que caracteriza a algunos materiales volcánicos retrabajados.

El análisis micromorfológico mostró que, no obstante que el adobe es un material tecnogénico, al que se le adicionaron diferentes componentes orgánicos e inorgánicos, aún sigue presentando evidencias de procesos de alteración que son propias de un suelo.

No obstante que las muestras estudiadas provienen de sitios geográficamente diferentes y con distintas temporalidades de ocupación, éstas muestran características y propiedades muy similares entre sí. Este hecho se considera indicativo de una continuidad en la técnica de su manufactura.

Para finalizar, es posible concluir que para obtener un mayor conocimiento teórico-práctico acerca del adobe, es necesario analizar diversas muestras provenientes de diferentes regiones culturales, de diferentes temporalidades $\mathrm{y}$ de diferentes geosistemas.

\section{Agradecimientos}

La investigación reportada en este artículo fue financiada con recursos de CONACYT (090220) otorgados a Jorge E. Gama Castro. Los autores agradecen a Annick Daneels, IIAUNAM y a Luis Fernando Guerrero Baca, coordinador de PROTERRA, por sus valiosos comentarios y sugerencias al manuscrito inicial. Asimismo, agradecen a Kumiko Shimada Miyasaka y Lucy Mora Palomino, quienes apoyaron en la realización de los análisis de laboratorio relativos a la determinación de carbono orgánico.

Así mismo se agradece al Dr. Héctor Javier Guzmán Olguín y al Ing. Gerardo Galguera Rosas el apoyo en la interpretación de algunas características físicas y mecánicas del adobe. Finalmente se agradece al biólogo Arturo Atilano Alvarado por su ayuda en la captura de información

\section{Referencias}

Almeida, M.R., 1998, La literatura gris: sistemas y redes en el ámbito nacional e internacional. Una propuesta para Brasil: Madrid, España, Universidad Complutense de Madrid, tesis doctoral, 263 p.
American Society for Testing and Materials (ASTM), 1985, Classification of Soils for Engineering Purposes: Philadelphia, Pennsylvania, American Society for Testing and Materials, 408 p.

Astrom, P., 1984, Excavations at Hala Sultan Tekke: Archaeology, 37(2), $58-59,77,81$

Barrera-Bassols, N., Zinck, J.A., 2003, Ethnopedology: a worldwide view on soil knowledge of local people: Geoderma, 111, 171-195.

Bouyoucos, G.J., 1963, Directions for making mechanical analyses of soils by the hydrometer method: Soil Science, 42, 225-230.

Braimoh, A.K., 2002, Integrating indigenous knowledge and soil science to develop a national soil classification system for Nigeria: Agriculture and Human Values, 19, 75-80.

Bullock, P., Fedoroff, N., Jongerius, A., Stoops, G., Tursina, T., Babel, U., 1985, Handbook for Soil Thin Section Description: Wolverhampton, U.K., Waine Research Publications, $152 \mathrm{p}$.

Cedeño, N.J., 1998, El culto al lugar central. Posibilidades en torno a un problema arqueológico: Arqueología, 20, 53-64.

Cervantes-Gutiérrez, V., Gama-Castro, J.E., Hernández-Cárdenas, G., Meave del Castillo, J.A., 2005, The land classification system of the San Nicolás Zoyatlan (S. Mexico) Nahuatl Indigenous community: a basis for a suitable parametric soil use proposal: Human Ecology Review, 12, 44-59.

Daneels, A., 1997, Settlement History in the Lower Cotaxtla Basin, en Stark, B.L., Arnold, P.J. (eds.), Olmec to Aztec Settlement Patterns in the Ancient Gulf Lowlands: Tucson, Arizona, The University of Arizona Press, 206-252.

Ericksen, P.J., Ardón, M., 2003, Similarities and differences between farmer and scientist views on soil quality issues in central Honduras: Geoderma, 111, 233-248.

Gama-Castro, J.E., Solleiro-Rebolledo, E., Vallejo-Gómez, E., 2000, Weathered pumice influence on selected alluvial properties in West Nayarit, Mexico: Soil \& Tillage Research, 55, 143-165.

Gama-Castro, J.E., Solleiro-Rebolledo, E., McClung, E., Villalpando, J.L., Sedov, S., Jasso-Castañeda, C., Palacios-Mayorga, S., Hernández, D., 2004, Contribuciones de la Ciencia del Suelo a la Investigación Arqueológica: El caso de Teotihuacan: Terra Latinoamericana, 23, $1-11$.

Gama-Castro, J.E., McClung de Tapia, E., Solleiro-Rebolledo, E., Ibarra, E., Sedov, S., Jasso-Castañeda, C., Vallejo-Gómez, E., Pi-Puig, T., Cabadas-Báez, H., 2005, Incorporation of ethnopedological knowledge in the study of soils in the Teotihuacan valley, Mexico: Eurasian Soil Science, 38, 95-98.

Gama-Castro, J.E., Solleiro-Rebolledo, E., Flores-Román, D., Sedov, S., Cabadas-Báez, H., Díaz-Ortega, J., 2007, Los tepetates y su dinámica sobre la degradación y el riesgo ambiental: el caso del Glacis de Buenavista, Morelos: Boletín de la Sociedad Geológica Mexicana, 59, 133-145.

Gee, G.W., Or, D., 2002, Particle-size analysis, en Dane, J. H. and Topp G.C. (eds.), Methods of Soil Analysis, Part 4: Physical Methods: Madison, Wisconsin, Soil Science Society of America, 255-293.

Gendrop, P., 2001, Diccionario de arquitectura mesoamericana: Ciudad de México, Trillas, $238 \mathrm{p}$.

Krasilnikov, P.V., 2002, Soil classifications and their correlation, en Krasilnikov, P.V. (ed.), Soil Terminology and Correlation: Petrozavodsk, Rusia, Karelian Research Centre, Russian Academy of Sciences, Institute of Biology, 7-41.

Krasilnikov, P.V., Tabor, J.A., 2003, Perspective on utilitarian ethnopedology: Geoderma, 111, 197-215.

López-Aguilar, F., 1991, Proyecto Valle del Mezquital: Informe de la cuarta temporada de campo: Ciudad de México, Archivo Técnico del Instituto Nacional de Antropología e Historia, informe técnico, $312 \mathrm{p}$.

López-Aguilar, F., 1994, Proyecto Valle del Mezquital: Informe de la quinta temporada de campo: Ciudad de México, Archivo Técnico del Instituto Nacional de Antropología e Historia, informe técnico, $154 \mathrm{p}$.

López-Aguilar, F., Solar Valverde, L., Vilanova, R., 1998, El Valle del Mezquital. Encrucijadas en la historia de los asentamientos humanos en un espacio discontinuo: Arqueología, 20, 21-40. 
López-Aguilar, F., Vilanova de Allende, R., Salvador G., 2007, Proyecto Específico Pahñú: Informe de la temporada 2007: Ciudad de México, Archivo Técnico del Instituto Nacional de Antropología e Historia, informe técnico, $153 \mathrm{p}$.

Munsell, 1975, Munsell soil color charts: Baltimore, Maryland, Macbeth Division of Kollmorgen Corporation, 34 p.

Natural Resources Conservation Service (NRCS), 2004, Soil Survey Laboratory Methods Manual: Lincoln, Nebraska, Natural Resources Conservation Service, United States Department of Agriculture, $735 \mathrm{p}$.

Organización de las Naciones para la Agricultura y la Alimentación (FAO), Centro Internacional de Referencia e Información en Suelos (ISRIC) y Sociedad Internacional de las Ciencias del Suelo (SICS), 1998, Base Referencial Mundial del Recurso Suelo: Roma, Italia, Organización de las Naciones Unidas para la Agricultura y la Alimentación, Centro Internacional de Referencia e Información en Suelos, Sociedad Internacional de las Ciencias del Suelo, Reporte 84,90 p.

Ramos-Galicia, Y., Hidalgo-Moreno, C., Sedov, S., Poetsch, T., 2003 Comales of Tzomantepec and paleosols: a case study: Revista Mexicana de Ciencias Geológicas, 20, 263-269.

Rivera-Torres, J.C., Muñoz-Díaz, E.E., 2005, Caracterización estructural de materiales de sistemas constructivos en tierra: el adobe: Revista Internacional de Desastres Naturales, Accidentes e Infraestructura Civil, 5, 135-148.

Sahagún, B., 1956, Historia general de las cosas de Nueva España, tomo III: Ciudad de México, Editorial Porrúa, 107 p.
Schoeneberger, P.J., Wysocki, D.A., Benham, E.C., Broderson, W.D. (eds.), 1998, Field Book for Describing and Sampling Soils: Lincoln, Nebraska, United States Department of Agriculture, Natural Resources Conservation Center, 125 p.

Soil Conservation Service (SCS), 1984, Procedures for collecting soil samples and methods of analysis for soil survey: Washington, D.C., Soil Conservation Service, United States Department of Agriculture, $68 \mathrm{p}$.

Solleiro-Rebolledo, E., Sedov, S., Gama-Castro, J.E., Flores-Román, D., Escamilla-Sarabia, G., 2003, Paleosol-Sedimentary sequences of the Glacis de Buenavista, Central Mexico: Interaction of Late Quaternary pedogenesis and volcanic sedimentation: Quaternary International, 106-107, 184-201.

Stoops, G., 2003, Guidelines for Analysis and Description of Soil and Regolith Thin Sections: Madison, Wisconsin, Soil Science Society of America, $184 \mathrm{p}$.

World Reference Base for Soil Resources (WRB), 2006, World Reference Base for Soil Resources 2006 (World Soil Resources Reports No. 103): Roma, Food and Agriculture Organization of the United Nations, 132 p.

Manuscrito recibido: Septiembre 8, 2010.

Manuscrito corregido recibido: Octubre 17, 2011

Manuscrito aceptado: Febrero 15, 2012. 